\title{
A Useful Family of Stochastic Processes for Modeling Shape Diffusions
}

\author{
Mousa Golalizadeh \\ Tarbiat Modares University
}

\begin{abstract}
One of the new area of research emerging in the field of statistics is the shape analysis. Shape is defined as all the geometrical information of an object whose location, scale and orientation is not of interest. Diffusion in shape analysis can be studied via either perturbation of the key coordinates identifying the initial object or random evolution of the shape itself. Reviewing the first case, we mainly consider the second case and particularly define a new family of diffusion processes. It can be used to model diffusion phenomena represented by shape evolution such as cell motion.
\end{abstract}

Keywords. Shape analysis; diffusion processes; shape coordinates; differential geometry; stationary distributions.

\section{Introduction}

In the ordinary language, the people are usually using more nominally familiar shape to describe the geometrical feature of an object. For example, it is known that the map of Iran looks like the shape of a cat. This is our subjective view of the shape whilst in order to analysis the shape of an object statistically we do need to quantify it in a proper manner. Before describing such procedure, let us first recall the formal definition of shape accepted between the statistical and mathematical communities.

The shape of the configuration is defined to be the geometrical information that remains when location, scale and rotation effects are removed out from an object Kendall (1984). It is seen that this clever definition justifies the concept of the shape verbally understood by most people. For instance, recalling our initial example, it does not matter how big the map of Iran 
is and where and in which orientation it is plotted - it it still 'cat-shaped'. Also, a typical version of standardization, frequently used in statistics, is seen in the formal definition of the shape. Recall that the standardization of a variable on one dimensional Euclidean space, taught and seen in the most elementary statistics courses and statistical books, is obtained by subtracting the variable from its mean (location parameter) and then dividing the result over the standard deviation (scale parameter) of the variable. It is known that by standardization one works with a new variable whose statistical features are either easy to derive or already well known in the statistical field. Similarly, in deriving the shape by omitting the translation, scale and orientation effects, known as Euclidean similarity transformations, from an object one is attempting to gather all objects under study in a common class. In another word, with these operations one will derive a set of the standardized shape data, ready to analysis statistically.

Having identified the shape of an object, one may be interested in studying the diffusion of the shape. Random evolution of the shape can be investigated through at least two different point of views. In the first view, the original points located on an object are assumed to perturb randomly in a Euclidean space. Note that the object, itself, is lied in a Euclidean space. Then, the properties of the shape of the object after an instant time point or in a limit are studied. It is obvious that this process is a continuous stochastic process in which the change of shape is described. This typical diffusion process is known as the diffusion of the Euclidean shape. D.G. Kendall, who was first to study the diffusion of shape, pointed out that this process generally is not Markovian, so not a diffusion, except in particular circumstance Kendall (1977). The exceptional case is in assuming the original diffusion to be Brownian. The interesting result in the diffusion of shape is given by D.G. Kendall stating that Brownian motion of the original points in the plane transforms to infinitesimally uncorrelated diffusion of size and shape, and the shape diffusion is Brownian motion specified directly in the shape space but with a random time change. Thereafter, there were many activities in this field by following the diffusion of Euclidean shape's point of view. See, for example, Kendall (1988), Kendall (1990), Le (1991) and Le (1994).

In the second point of view, the random behavior of the shape itself, rather than the original points located on the object, is considered as the source of randomness. That is a study on a diffusion in the shape space. We shall see below that the shape space is in fact a non-Euclidean space, so the 
diffusion process, and generally the stochastic process, in the shape would be a typical one in the field of statistics. Although it is expected to consider the motion of the whole shape, the common procedure in this situation is to assume that the coordinates of the shape lied in the shape space are perturbing randomly (see, e.g. Le, 1994, Kendall, 1998, and Kendall, 1990). Assuming this sort of diffusion, one may be interested in many statistical features of the shape diffusion such as the stationary distribution of a random process proposed in the shape space. Note that because the shapes can be represented on manifolds Small (1996), one can also study the stochastic processes on a Riemannian manifold (see, e.g. Ikeda and Wanatabe, 1981) and then consider the special cases of which the shape space is constructed. This approach is worth in theoretical investigations, while in the application studies the former approach is more popular.

In the subsequent sections, we first describe some preliminary materials useful for the rest of the paper in the Section 2. It includes describing two shape coordinate systems and a brief review of the diffusion on manifolds. Then, we consider the diffusion processes on the shape space in the Section 3. Particularly, we pay attention to an important process suitable to model shape evolution phenomena in our real life such as cell motion. The stationary distribution of the proposed process, inference about the involved parameters and a simulation study are also presented in this section. The paper is ended with conclusion along with direction to the future work.

\section{Preliminaries}

In this section we provide the required materials for the subsequent sections. For the purpose of this paper, we use the notations implemented in Dryden and Mardia (1998). Also, following the formal definition of the shape and for consistency, the procedure to obtain the shape of an object is describe based on the method implemented by Kendall (1984). It is known as the Kendall coordinate system. However, in order to present the new results in this paper we also use another shape coordinate system.

\subsection{Shape Coordinate Systems}

Recalling the shape definition, one can see that many mathematical concepts, operations and functions are involved in the process of obtaining the shape of an object. Before highlighting them let us, first, study the procedure to 
quantify an object. A common practical approach is to locate a set of points on each object. The finite number of the points located on an object which represent its key feature are called landmarks. There are three various types of landmarks known as mathematical, anatomical and pseudo landmarks (see, Dryden and Mardia, 1998, pp. 2-5). It is common to locate landmarks on the outline of an object, although the researcher can also set them in the interior body of the object. However, for the aim of this paper the later case does not provide us any further information and so is not considered here.

Since the object are usually lying in two or three dimensional space, the position coordinates of a landmark on each object are represented as pairs of $x$ and $y$ or triplet $x, y$ and $z$. In this article only two dimensional objects are considered. Further, the case in which all landmarks are concentred in one single point is not of interest.

Consider a configuration of $k \geqslant 3$ points in two dimensions. The configuration matrix, say $X$, is the $k \times 2$ matrix of Cartesian co-ordinates of the $k$ landmarks in two dimensions, i.e.,

$$
\boldsymbol{X}^{T}=\left[\begin{array}{llll}
x_{1} & x_{2} & \ldots & x_{k} \\
y_{1} & y_{2} & \ldots & y_{k}
\end{array}\right] \text {. }
$$

Usually, the first step in obtaining the shape is to remove the location information by pre-multiplying $X$, with Helmert sub-matrix $H$, which is the $(k-1) \times k$ Helmert matrix without the first row. We write $X_{H}=H X$ as the Helmertized matrix. Then, the scale is removed by dividing by $\left\|X_{H}\right\|=$ $\|H X\|$ to get the pre-shape of $X$. Finally, the shape is obtained via postmultiplying the pre-shape by a rotation matrix, say $\Gamma$. Note that if one does not remove the scale effect what is left is known as "size-and-shape" of $X$.

Since points in two dimensions can be represented with the complex number, we can identify a landmark with coordinate, say $\left(x_{j}, y_{j}\right), j=1, \ldots, k$, located on an object as $z_{j}^{\circ}=x_{j}+i y_{j}$. Then, considering the original complex landmarks $z^{\circ}=\left(z_{1}^{\circ}, z_{2}^{\circ}, \ldots, z_{k}^{\circ}\right)^{T}$, and the Helmertized landmarks $z_{H}=$ $H z^{\circ}=\left(z_{1}, z_{2}, \ldots, z_{k-1}\right)^{T}$, the shape of $X$ can be identified by coordinates $\left(w_{1}, w_{2}, \ldots, w_{k-2}\right)^{T}$, where $w_{j}=u_{j}+i v_{j}=\frac{z_{j}}{z_{k-1}}$. These coordinates are known as the Kendall coordinate systems in the literature and are usually identified by the superscript $K$ in $u_{j}$ and $v_{j}$ 's, i.e. $w_{j}^{K}=u_{j}^{K}+i v_{j}^{K}$. Note that it is convenient to represent the shape of $X$ by the real $(2 k-4)$-vector

$$
u^{K}=\left(u_{1}^{K}, u_{2}^{K}, \ldots, u_{k-2}^{K}, v_{1}^{K}, v_{2}^{K}, \ldots, v_{k-2}^{K}\right)^{T} .
$$

The new results in this paper is based upon the Kent polar coordinate systems Kent (1994). Hence, let us define the procedure to derive it here. 
Using the Helmertized landmarks, consider the transformation $z_{j}=s_{j}^{\frac{1}{2}} e^{i \theta_{j}}$, where $s_{j} \geqslant 0, \quad 0 \leqslant \theta_{j} \leqslant 2 \pi$ and $\sum_{i=1}^{k-1} s_{i}=1$. Then, the Kent polar coordinates for the shape of $X$ are $\left(\boldsymbol{S}^{T}, \boldsymbol{\theta}^{T}\right)^{T}=\left(s_{1}, \ldots, s_{k-2}, \theta_{1}, \ldots, \theta_{k-2}\right)^{T}$. Let us write $D_{s}=\operatorname{diag}\left(\sqrt{s_{1}}, \ldots, \sqrt{s_{k-2}}\right)$ and $\gamma(s)=1-\sum_{i=1}^{k-2} s_{i}$, to simplify the further computations. Note that transformation between this and other shape coordinate systems including the Kendall coordinates is straightforward (see, e.g. Dryden and Mardia, 1998).

It is worth here to review some geometrical properties of the shape space. The shape space of $k$ points in $m$ dimensions, located on the outline of an object, is denoted by $\Sigma_{m}^{k}$. Due to loosing $m$ dimensions for location, one dimension for scale and $\frac{1}{2} m(m-1)$ dimensions for rotation, the shape space is $k m-m-1-\frac{m(m-1)}{2}$ dimensions. Note that the size-and-shape space is written as $S \Sigma_{m}^{k}$, which is a $k m-m-\frac{m(m-1)}{2}$ dimensional space.

Special cases of the shape space for the two dimensional shapes is of interest. In two dimensional case the shape space is the complex projective space with sectional curvature 4 and particularly for triangles in a plane it is the sphere in three dimensions with radius $\frac{1}{2}$, Kendall (1984). It is clear that the shape space is a non-Euclidean space because not only the shape space $\Sigma_{2}^{k}, k>3$, i.e. the complex projective space, but also the sphere, which is the shape space of 3 points in two dimensions, are non-Euclidean spaces. It should be noted that the metric considered by Kendall to study the distance between shapes is the Procrustes distance, which is one of the suitable Riemannian metrics to compare objects in non-Euclidean space.

\subsection{Diffusion Processes on Manifolds}

As was mentioned, the shape space is an non-Euclidean space. So, since the shape spaces contains the curved surfaces, known as a manifold, the methods of differential geometry can be used to represent shapes. Hence, to understand the diffusion processes in the shape space one can study them first on manifolds.

Many important operations in differential geometry can be transferred to a class of stochastic curves using stochastic calculus. Then, using a class of affine connections one can obtain diffusions on a manifold. Precisely, the procedure involves constructing a flow of a diffeomorphism on the bundle of orthonormal frames over the manifold Ikeda and Wanatabe (1981), p. 261. In addition, a non-singular diffusion induces a Riemannian structure on manifold where the elements of the structure are generated by diffusing 
local coordinates (see, e.g. Rogers and Williams, 1987, pp. 203-224).

The shape space is usually identified by local coordinates. They are known as the shape coordinate system, which are simply the result of various mathematical approaches to obtain the shape. Closely related to them are the Riemannian metric induced by the infinitesimal changes of the coordinates along a path. On the other hand, having the differential operators in a manifold one can derive the infinitesimal operators of a diffusion process (see, e.g. Meyer, 1966, pp. 256-270). One of the key tools useful to obtain the infinitesimal parameters of a diffusion process on a Riemannian manifold is the Laplace-Beltrami operator. It is used to define the Brownian motion in both shape and size-and-shape spaces Kendall, et al. (1999). Note that the Laplace-Beltrami operator is usually defined as "div grad" in the stochastic textbooks.

Among many diffusion processes the Brownian motion and OrnsteinUhlenbeck (OU) processes are of great important for both probabilists and statisticians. For example, in studying the motion of a particle, the Brownian motion only describes its position while the OU process explains its velocity as a function of time as well. In the shape analysis these processes can be used to model the random evolution of geometrical objects such as cell motion during a time period.

\section{Diffusions on Shape Space}

The initial work in the subject of the diffusion of shape is done by Kendall (1977). He has explored many aspects of diffusion of $k$ particles in one and two dimensions. He has noted that although a diffusive motion for $k \geqslant 3$ particles in $m \geqslant 1$ will result in a continuous stochastic process of shape, it will not be a Markovian process. However, assuming Brownian motion for the original landmarks he proved that the resulting shape is also Brownian but with respect to the random time change. His son has explored this statement and other relevant results via symbolic computation Kendall (1988). He has further given important results for the both Brownian and OU processes using various shape coordinates Kendall (1990).

Based on the Riemannian metric tensor in $\Sigma_{2}^{k}$ using Kendall coordinate system, Ball and his colleagues derived the Brownian motion specified directly in the planar shape space Ball, et al. (2008). They further identified the infinitesimal parameters of the Brownian motion represented by an stochastic differential equation (SDE) and introduced a family of OU pro- 
cesses on $\Sigma_{2}^{k}$. Then, having the simple form of the SDEs for both processes, they described the procedure to simulate the random shapes moving towards a reference shape and in particular to model cell motion. As a motivation to analysis the cell motions in further details, we define a new family of OU processes in which its counterpart Brownian motion has the uniform shape density as its stationary distribution.

\subsection{New OU Processes in Two Dimensional Shape Space}

By adding an extra drift to the standard Brownian motion using Kent coordinate proposed in Ball, et al. (2008), we can define a new family of OU processes on $\Sigma_{2}^{k}$. The new process is defined by the following SDEs

$$
\begin{aligned}
\mathrm{d} \mathbf{S}= & {\left[-4 \kappa \gamma(s) \mathbf{S}+2\left\{1_{k-2}-(k-1) \mathbf{S}\right\}\right] \mathrm{d} t } \\
& +2\left\{-\frac{D_{s}^{2} 1_{k-2} 1_{k-2}^{T}}{1+\sqrt{\gamma(s)}}+I_{k-2}\right\} D_{s} \mathrm{~d} B_{t}^{(s)}, \\
\mathrm{d} \boldsymbol{\theta}= & \frac{1}{\sqrt{\gamma(s)}}\left\{\frac{1_{k-2} 1_{k-2}^{T} D_{s}}{1+\sqrt{\gamma(s)}}+\sqrt{\gamma(s)} D_{s}^{-1}\right\} \mathrm{d} B_{t}^{(\theta)},
\end{aligned}
$$

where $\kappa>0$ is a constant. The first expression added to the drift function indicates the strength of the infinitesimal drift towards the reference shape with the Helmertized coordinates $\mu_{0}=(1,0, \ldots, 0)^{T}$. Further, comparing the first equation in (2) with the SDE of the standard Brownian motion using Kent coordinate given in Ball, et al. (2008) shows that the term $-4 \kappa \gamma(s) \mathbf{S}$ is analogous to the drift of an OU process on the line, say $\mathrm{d} X_{t}=-\kappa X_{t} \mathrm{~d} t+$ $\mathrm{d} B_{t}$. The OU processes given by (2), also, possess the important feature of independency of polar coordinates $\mathbf{S}$ and $\boldsymbol{\theta}$. This property would be very useful in where one is interested on simulating the processes. In other words, in simulating the discretized version of the SDEs (2), two random processes can be generated independently, each having its own criteria. Further, since $z_{k-1} \neq 0$ the term $\gamma(s)$ is not zero and so the above SDEs are defined everywhere.

Note that the SDEs here are in Itô form (see, e.g. Karlin and Taylor (1981). Also, since the shape space $\Sigma_{2}^{k}$ is homogeneous the reference shape can be any particular shape rather than the point $\mu_{0}=(1,0, \ldots, 0)^{T}$. That shape can be transferred to the reference shape $\mu_{0}$ using a suitable unitary transformation and the other new coordinates will be obtained accordingly. 
This feature is of great importance in which the reference shape is unknown and one is going to follow a statistical procedure to estimate it.

Having considered the cell motion as a shape diffusion, both geometrical and statistical aspects of this evolution can be investigated by the above modelling. For instance, if it is assumed that cells are moving, with a smooth drift, towards a particular cell but subject to a random fluctuation of their positions the above SDEs can handle this feature. An simulating study dealing with cell motions is discussed later. But we, first, provide a graphical example to motivate the latter simulation study.

Initial motivation behind the considered OU process came from an analysis of cell motion by Ionides (2001). Ionides has studied the movements of ten cells recorded at seven equally spaced time points using some predefined SDEs. However, whole geometric aspect of motion has not been taken into account in his work. In other words, the positions of landmarks on the outline of cells, as the main source of the information to do a statistical (labeled) shape analysis, were not used to model the movement. We expect the shape of cells at any time instant, considered by Ionides (2001), is the result of movement of so many points which are set at the outline of a unit circle. Hence, our OU process could capture these feature. As an example, the circle in the Figure 1 is the initial shape (cell) generated by our OU process with the parameters fixed as $k=100, \kappa=100$, and $\Delta t=0.005$.

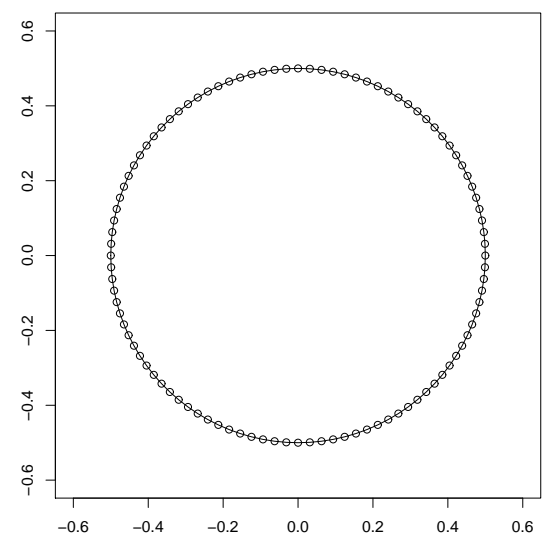

Figure 1. Circle with landmarks on its outline. The circle is the initial shape in a sequence of shape data generated by the SDEs in (2). The parameters are fixed as $k=100, \kappa=100$, and $\triangle t=0.005$. It is expected the perturbation of the landmarks would produce new objects which is somewhat similar to the shape of cells considered by Ionides (2001). 


\subsection{Stationary Distributions}

Having defined some continuous stochastic processes, one can derive their limiting behaviors leading to stationary distributions. For the one dimensional stationary diffusion processes an explicit formula to derive the stationary distribution is available (see, e.g. Karlin and Taylor, 1981, p. 221). However, there is not a closed form for the multidimensional case. To overcome this one can turn around the problem. If a stationary distribution exists it necessarily satisfies the Kolmogorov forward equation of the diffusion process under study. Hence, for a given distribution one can verifies whether or not it is satisfies the Kolmogorov forward equation of the corresponding multidimensional stochastic process. If the answer is YES, the distribution is in fact the stationary distribution. Although this method involves tedious mathematical calculations, it is quite helpful. Particularly, many involved manipulations can be handled by the available computer packages such as Maple. We followed this procedure for our proposed OU process for multidimensional cases. Recall that a stationary diffusion process $X_{t}$ with drift and motion $\mu$ and $\boldsymbol{\sigma}$, respectively, and stationary distribution $f$ satisfies in the Kolmogorov forward equation

$$
\sum_{i=1}^{n} \frac{\partial\left[\mu_{i}(x) f\right]}{\partial x_{i}}-\frac{1}{2} \sum_{i, j=1}^{n} \frac{\partial^{2}\left[\left(\boldsymbol{\sigma} \boldsymbol{\sigma}^{T}\right)_{i j}(x) f\right]}{\partial x_{i} \partial x_{j}}=0 .
$$

One of the important distributions in the statistical shape analysis is the uniform distribution. The uniform density with respect to Lebesgue measure using Kendall coordinate system is given as

$$
\frac{(k-2) !}{\pi^{k-2}}\left\{1+\left(u^{K}\right)^{T} u^{K}\right\}^{1-k} .
$$

This distribution using Kent coordinate system is $(k-2) ! /(2 \pi)^{k-2}$. So, it is more natural to use this coordinate systems to represent the uniform distribution in the shape space $\Sigma_{2}^{k}$ as analogous with the uniform distribution on the line. The interesting point is that if the original landmarks are independently identically distributed with a rotationally symmetric distribution, then the resulting shape distribution is uniform in the shape space (see, e.g. Dryden and Mardia, 1998, p. 111). Ball and his colleagues have shown that the uniform distribution $(k-2) ! /(2 \pi)^{k-2}$ is the invariant distribution of the standard Brownian motion on $\Sigma_{2}^{k}$ using the Kent polar coordinates Ball, et al. (2008). 
The other shape distributions for two dimensional data include the complex Bingham, complex Watson, complex angular central Gaussian and offset normal distributions (see, e.g. Dryden and Mardia, 1998, Chapter. 6). It is of interest to verify whether or not any of them is the invariant distribution for a family of OU processes on $\Sigma_{2}^{k}$. Ball and his colleagues have defined a family of OU processes in which the shapes are moving towards a particular reference shape Ball, et al. (2008). They have shown that for particular situation the stationary distribution of their OU processes is the complex Watson distribution, written as

$$
f\left(u^{K}\right)=\frac{(k-3) !\left\{1+\left(u^{K}\right)^{T} u^{K}\right\}^{1-k}}{2 \pi^{k-2} C_{2}(\kappa)} \cdot \exp \left\{\kappa \frac{1-\left(u^{K}\right)^{T} u^{K}}{1+\left(u^{K}\right)^{T} u^{K}}\right\},
$$

where

$$
C_{2}(\kappa)=\frac{(k-3) ! \mathrm{e}^{-\kappa}}{\kappa^{k-2} 2^{k-1}}\left\{\mathrm{e}^{2 \kappa}-\sum_{r=0}^{k-3} \frac{(2 \kappa)^{r}}{r !}\right\}=\frac{\mathrm{e}^{-\kappa}}{2(k-2)}{ }_{1} \mathrm{~F}_{1}(1 ; k-1 ; 2 \kappa),
$$

where

$$
{ }_{1} F_{1}(a ; b ; x)=1+\frac{a}{b} \frac{x}{1 !}+\frac{a(a+1)}{b(b+1)} \frac{x^{2}}{2 !}+\frac{a(a+1)(a+2)}{b(b+1)(b+2)} \frac{x^{3}}{3 !}+\cdots
$$

is the confluent hypergeometric function.

In order to obtain the complex Watson distribution represented by the Kent coordinate system, we could, first, derive its corresponding distribution in terms of Kendall polar coordinate. That can be implemented via the equality $u_{j}^{K}+i v_{j}^{K}=r_{j} e^{i \phi_{j}}, j=1, \ldots, k-2$, with the Jacobian $\prod_{j=1}^{k-2} r_{j}$, yielding

$$
\begin{aligned}
f\left(r_{1}, \ldots, r_{k-2}, \phi_{1}, \ldots, \phi_{k-2}\right)= & \frac{(k-3) !\left\{+\sum_{j=1}^{k-2} r_{j}^{2}\right\}^{1-k} \prod_{j=1}^{k-2} r_{j}}{2 \pi^{k-2} C_{2}(\kappa)} \\
& \times \exp \left\{\begin{array}{c}
1-\sum_{j=1}^{k-2} r_{j}^{2} \\
\kappa-\sum_{j=1}^{k-2} r_{j}^{2}
\end{array}\right\},
\end{aligned}
$$


Then, we could utilize the relationship between Kendall and Kent coordinates (both in polar systems) to get the required distribution. It is known that (see, e.g. Dryden and Mardia, 1998, P. 110) $\phi_{j}=\left(\theta_{j}-\theta_{k-1}\right) \bmod 2 \pi$, and

$$
r_{j}^{2}=\frac{s_{j}}{\left(1-\sum_{j=1}^{k-2} s_{j}\right)}, \quad j=1, \ldots, k-2 .
$$

and so $\left|\partial s_{j} / \partial r_{j}\right|=2^{k-2}\left\{1+\sum_{j=1}^{k-2} r_{j}^{2}\right\}^{1-k} \prod_{j=1}^{k-2} r_{j}$.

On substituting (7) into (6) and considering the above Jacobian, the complex Watson distribution represented by the Kent coordinate system, with respect to Lebesgue measure, turns out as

$$
f(\mathbf{S}, \boldsymbol{\theta})=\frac{(k-3) !}{2^{k-1} \pi^{k-2} C_{2}(\kappa)} \cdot \exp \left\{\kappa\left(1-2 \sum_{i=1}^{k-2} s_{i}\right)\right\},
$$

As can be seen, the random variables $\mathbf{S}$ and $\boldsymbol{\theta}$ are independent with $f(\boldsymbol{\theta})=$ $1 /(2 \pi)^{k-2}$. This fact will help us to verify whether or not this distribution is the stationary distribution of our new OU process given by the SDEs (2) while invoking the Kolmogorov forward equation given by (3).

To have an idea in how to verify if the distribution in (8) is the stationary distribution of the OU process with the SDEs (2), let us consider the case $k-2=1$, i.e. three points in two dimensions. The procedure for other cases, i.e. $k \geqslant 4$ will be quite similar but with more complexity due to very troublesome calculations. Recalling the equation (2), the SDE of our OU process for $k=3$ will break down to

$$
\begin{aligned}
\mathrm{d} S & =\{-4 \kappa S(1-S)+2(1-2 S)\} \mathrm{d} t+2\left(-\frac{S}{1+\sqrt{1-S}}+1\right) \sqrt{S} \mathrm{~d} B_{t}^{S} \\
& =\mu(S) \mathrm{d} t+\sigma(S) \mathrm{d} B_{t}^{S}, \\
\mathrm{~d} \theta & =\frac{1}{\sqrt{1-S}}\left(\frac{\sqrt{S}}{1+\sqrt{1-S}}+\frac{\sqrt{1-S}}{\sqrt{S}}\right) \mathrm{d} B_{t}^{\theta}=\sigma(\theta) \mathrm{d} B_{t}^{\theta},
\end{aligned}
$$

where $\left(B_{t}^{S}, B_{t}^{\theta}\right)$ is 2-dimensional standard Brownian motion. In this particular case, the distribution in (8) turns out to

$$
\begin{aligned}
f(S, \theta) & =\frac{1}{2 \pi} \cdot \frac{1}{2 C_{2}(\kappa)} e^{\kappa(1-2 S)}=\frac{1}{2 \pi} \cdot \frac{2 \kappa}{e^{\kappa}-e^{-\kappa}} e^{\kappa(1-2 S)} \\
& =f(\theta) f(S), \quad 0 \leqslant \theta \leqslant 2 \pi, \quad 0 \leqslant s \leqslant 1 .
\end{aligned}
$$


Using these information and the independency of the random processes $S$ and $\theta$, the left hand side of the Kolmogorov forward equation in (3) is also simplified as

$$
\frac{\partial[\mu(S) f(S)]}{\partial S}-\frac{1}{2} \frac{\partial^{2}\left[\sigma^{2}(S) f(S)\right]}{\partial S^{2}} .
$$

Ignoring the normalising constants of the marginal distribution $f(S)$, this final expression can be rewritten as

$$
\frac{\partial\left[\{-4 \kappa S(1-S)+2(1-2 S)\} e^{\kappa(1-2 S)}\right]}{\partial S}-\frac{1}{2} \frac{\partial^{2}\left[4 S(1-S) e^{\kappa(1-2 S)}\right]}{\partial S^{2}} .
$$

Note that none of the infinitesimal parameters in the SDEs in (9) and quantities in joint distribution in (10) are involved in $\theta$, and so their derivatives with respect to $\theta$ are zero. Consequently, as expected, no expression of $\theta$ appears in the Kolmogorov forward equation for the stochastic process of $(S, \theta)$. Now, we should check whether or not the expression in (11) is equal to zero. That is a matter of differentiation and could be easily shown that the final result is indeed zero. Hence, the distribution in (10) is the stationary density of our proposed OU process with the SDEs given in (9).

The complexity of mathematical calculations involved in deriving SDEs, stationary distributions and Kolmogorov forward equations in studying diffusion processes for more points, i.e. $k \geqslant 4$ can be seen from the above particular case. We used arithmetic to break down the rigorous manipulations for other scenarios, i.e. $k \geqslant 4$. The results were all promising in both delivering the SDEs in (2) and distributions in (8) and, further, evaluating the equality in (3) to justify that the complex Watson distribution is the stationary distribution of our OU process.

\subsection{Inference}

In real applications, the parameter $\kappa$ is usually unknown. Here, we only consider the classical statistics approach, specifically the pseudo maximum likelihood method, to derive an estimate for $\kappa$. The other statistical inference methods will be our future topic. Note that other parameters, i.e. the reference shape and the variance of the time instance, can be unknown as well. However, for the purpose of this paper, we assume that the only unknown parameter is the strength of the drift, i.e. $\kappa$, for the proposed OU with SDEs given in (2) or equivalently the concentration parameter of the distribution in (8). 
Suppose a sample of size $n$ shape data identified by the shape coordinate systems $(\mathbf{S}, \boldsymbol{\theta})$, are modeled by distribution (8) and the aim is to derive a maximum likelihood estimator for $\kappa$. Note that making the inference just based upon this distribution means that the shape data collected over time are independent sample. However, our data, generated from the OU process (2), are highly correlated. Nevertheless, we can assume that the available are from stationary distribution with known reference shape $\mu_{0}$. Let us $D=\left\{\left(\mathbf{S}_{1}, \boldsymbol{\theta}_{1}\right),\left(\mathbf{S}_{2}, \boldsymbol{\theta}_{2}\right), \ldots,\left(\mathbf{S}_{n}, \boldsymbol{\theta}_{n}\right)\right\}^{T}$, indicates the shape data. Then, the logarithm of the likelihood of $\kappa$ up to constants is

$$
\log L(\kappa \mid D)=l(\kappa)=\kappa\left(n-2 \sum_{i=1}^{n} 1_{k-2}^{T} \mathbf{S}_{i}\right)-n \log C_{2}(\kappa),
$$

where $1_{q}$ is the column $q$-vector of ones. Setting the derivative, with respect to $\kappa$, of this last equation to zero and assuming $C_{2}^{\prime}(\kappa)=\frac{\partial C_{2}(\kappa)}{\partial \kappa}$, gives the MLE of $\kappa$ as the solution of the following equality

$$
\frac{C_{2}^{\prime}(\kappa)}{C_{2}(\kappa)}=1-2 \frac{\sum_{i=1}^{n} 1_{k-2}^{T} \mathbf{S}_{i}}{n} .
$$

If it is known that the concentrations parameter $(\kappa)$ is too big, we can use the approximate equality

$$
C_{2}(\kappa) \approx \frac{(k-3) !}{\kappa^{k-2} 2^{k-1}} e^{\kappa}
$$

and so (see, e.g. Dryden and Mardia, 1998, P. 121)

$$
C_{2}^{\prime}(\kappa) \approx \frac{(k-3) !}{\kappa^{k-2} 2^{k-1}} e^{\kappa}\left[1-\frac{k-2}{\kappa}\right] .
$$

Plugging these information into the equation (12), gives the approximate MLE for high concentration $\kappa$, as $\hat{\kappa} \approx n(k-2) / 2 \sum_{i=1}^{n} 1_{k-2}^{T} \mathbf{S}_{i}$.

\subsection{A Simulation Study}

In this section, we provide a simulation study to generate shape data, to model the random feature of them and to make inference about a concentration parameter. In fact, the simulation study is proposed to mimic the behavior of cell motion Ionides (2001). The procedure is as follows. 
We started with initial circle given in Figure 1 and, then, the subsequent shape data (cells) were generated using the OU process given in (2) with the parameters fixed as in Figure 1. In total we had 1000 shape data. Because the shape data in terms of Kent polar coordinates lie in non-Euclidean space they cannot be visualized well on a computer screen or a piece of paper. Hence, for plotting purpose, all shapes were transferred to the Bookstein coordinate system using simple relationship between shape coordinates (see, e.g. Dryden and Mardia, 1998). As an example, Figure 2 shows the shape figure of the objects at some time instants. Since the time scale is too small the difference between shapes are not obvious. However, it moves well towards the reference shape with just altering the concentration parameters. Note that in this example we assumed that the reference shape is known a priory as $\mu_{0}$. The estimate of $\kappa$ given by approximate MLE led to the value 129 which is far from the actual value. However, this may be due to both ignoring the high dependency of generated data and fixing the other parameters.
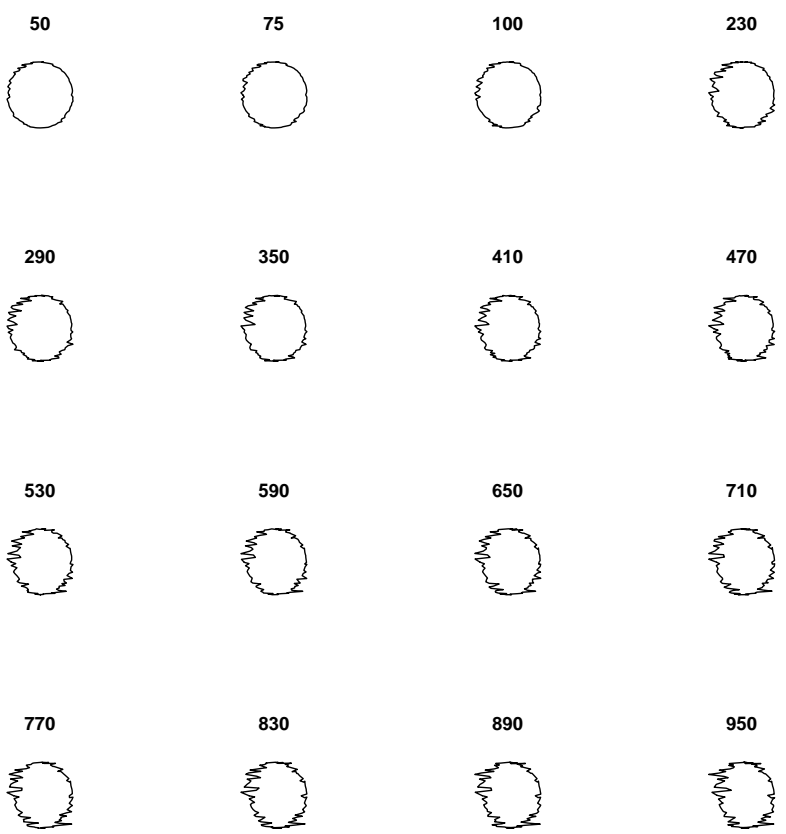

Figure 2. Shape of some data generated by the SDEs in (2). The plot shows the shape of objects at some time instants with the parameters fixed as $k=100, \kappa=100$, and $\triangle t=0.005$. It mimics the diffusions of cells considered by Ionides (2001). 
To consider the correlation around the outline, as treated by Kent and his colleagues Kent, et al. (2000), may describe the real diffusion of cells much better than the case studied here. It will be investigated in future research.

\section{Conclusion}

In studying the random behavior of an object, perturbation of individual points cannot give any insight about the next state of the geometrical structure of entire object. To take into account whole object, as data observation, and study diffusion of its shape in the space, where it lies, could provide more information in making inference, doing prediction and further statistical analysis about object itself rather than the individual points. The paper introduced a new family of stochastic process for modeling shape diffusion. It was used to model cell diffusion where the cells are moving towards particular shape. The SDEs on the process were presented and the stationary distribution was derived. The statistical inference about the concentration parameter through maximum likelihood method was made and a simulation study was carried out to both mimic the cell motion and have an estimate for the parameter. Due to nature of modeling, it is more feasible to consider the other statistical methods to estimate concentration and other involved parameters. Applying the proposed processes in some real data will definitely arise many other challenges dealing with applied shape analysis which are worth to study in future.

\section{References}

Ball, F.G., Dryden, I.L. and Golalizadeh, M. (2008). Brownian motion and OrnsteinUhlenbeck processes in planar shape space, Methodology and Computing in Applied Probability, 10, 1-22.

Dryden, I.L. and Mardia, K.V. (1998). Statistical Shape Analysis. John Wiley and Sons, Chichester.

Ikeda, N. and Wanatabe, S. (1981). Stochastic Differential Equations and Diffusion Processes. North-Holland, Amsterdam.

Ionides, E.L. (2001). Statistical Analysis of Cell Motion. Ph.D. thesis, University of California, Berkeley.

Karlin,S. and Taylor, H.M. (1981). A Second Course in Stochastic Processes. Academic Press, New York. 
Kendall, D.G. (1977). The diffusion of shape, Advances in Applied Probability, 9, 428-430.

Kendall, D.G. (1984). Shape manifolds, procrustean metrics and complex projective spaces, Bulletin of the London Mathematical Society, 16, 81-121.

Kendall, D.G., Barden, D., Carne, T.K. and Le, H. (1999). Shape and Shape Theory. John Wiley and Sons, Chichester.

Kendall, W.S. (1988). Symbolic compution and the diffusion of triads, Advances in Applied Probability, 20, 775-797.

Kendall, W.S. (1990). The Diffusion of Euclidean Shape. in: Grimmett, G. and Welsh, D. eds. Disorder in Physical Systems, Oxford University Press., Oxford.

Kendall, W.S. (1998). A diffusion model for Bookstein triangle shape, Advances in Applied Probability, 33, 317-334.

Kent, J.T. (1994). The complex bingham distribution and shape analysis, Journal of the Royal Statistical Society. Series B, 56, 285-299.

Kent, J.T., Dryden, I.L. and Anderson, K. R. (2000). Using circulant symmetry to model featureless objects, Biometrika, 87, 527-544.

Le, H. (1991). A stochastic calculus approach to the shape distribution induced by a complex normal model, Mathematical Proceedings of the Cambridge Philosophical Society, 1091, 221228.

Le, H. (1994). Brownian motion on shape and size-and-shape spaces, Journal of Applied Probability, 31, 101-113.

Meyer, P.A. (1966). Probability and Potentials. Blaisdell, Mass.

Rogers, L. and Williams, D. (1987). Diffusion, Markov processes, and Martingales, Volume 2, Itô calculus. John Wiley and Sons, UK.

Small, C.G. (1996). The Statistical Theory of Shape. Springer-Verlage, New York.

\section{Mouse Golalizadeh}

Department of Statistics,

Faculty of Mathematical Science,

Tarbiat Modares University,

Tehran, Iran.

email: golalizadeh@modares.ac.ir 